\title{
In Vivo Derm Dotting: An Easy and Inexpensive Way for Clinicopathological Correlation
}

\author{
Sven Lanssens ${ }^{1}$, Katrien Vossaert ${ }^{1}$, Sofie De Schepper ${ }^{2}$
}

1 Dermatologie Maldegem, Maldegem, Belgium
2 University Hospital Ghent, Department of Dermatology, Ghent, Belgium

Key words: clinicopathological correlation, derm dotting, dermoscopy, nail polish

Citation: Lanssens S, Vossaert K, De Schepper S. In vivo derm dotting: an easy and inexpensive way for clinicopathological correlation. Dermatol Pract Concept. 2019;9(1):71-72. DOI: https://doi.org/10.5826/dpc.0901a17

Published: January 31, 2019

Copyright: $@ 2019$ Lanssens et al. This is an open-access article distributed under the terms of the Creative Commons Attribution License, which permits unrestricted use, distribution, and reproduction in any medium, provided the original author and source are credited.

Funding: None.

Competing interests: The authors have no conflicts of interest to disclose.

Authorship: All authors have contributed significantly to this publication.

Corresponding author: Sven Lanssens, MD, Dermatologie Maldegem, Stationsstraat 929990 Maldegem, Belgium. Email: Sven.Lanssens@ gmail.com

\section{Introduction}

In 2013, ex vivo derm dotting was published as a technique to mark a dermoscopically interesting area by dotting it with nail polish after fixation of the specimen [1]. Although interesting, this technique may have its disadvantages. First, the pathologist needs to know dermoscopy. Second, we already have shown that ex vivo and in vivo dermoscopic images display different features: some structures and colors are less clear or even lost, while others become more prominent [2]. This may lead to different areas of interest in vivo and ex vivo. Moreover, pathologists have been using the technique for only a few years, and they are still developing criteria for it.

\section{Case Presentation}

Dermatologists are trained dermoscopists and know the areas of highest interest (eg, ulceration in melanoma, changed area in existing nevus, blood vessel alterations, and collisional lesions). Hence we tried to perform derm dotting in vivo by placing the nail polish dot before excision and processing. The dot survived transportation in formalin and the different processing stages in the laboratory (formalin 10\% fixation for 48 hours, paraffin embedding, cutting, and hematoxylin and eosin staining or immunohistochemical staining). The dot remained in place, which enabled us to make a pathology slide through it. The marked area remained easily detectable and visible under the microscope (Figure 1).

To fine-tune the practical aspects, we applied different types, sizes, and colors of nail polish to pig skin.

Applying the nail polish drop with a toothpick or a nail art brush was most rewarding. The thicker drops created using the commercial brush covered bigger areas than intended and detached easily during processing. Smaller drops were easily discernable by the laboratory technician and under the microscope. Their quicker drying times also allowed us to check dermoscopically in vivo to determine whether the dot was accurately placed. Red, orange, blue, and black yielded the best visibility. Multiple colors in one specimen are possible for collisional lesions or multiple areas of interest (Figure 2). 


\section{Conclusions}

We conclude in vivo derm dotting is an easy and inexpensive method for clinicopathological correlation, and we believe it should be incorporated in routine dermatological practice. All tested commercially available nail polishes worked perfectly, but small drops of red, orange, blue, and black yielded the best results.

\section{References}

1. Haspeslagh M, Degryse N, De Wispelaere I. Routine use of ex vivo dermoscopy with "derm dotting" in dermatopathology. Am J Dermatopathol. 2013;35(8):867-869.

2. Haspeslagh M, Vossaert K, Lanssens S, et al. Comparison of ex vivo and in vivo dermoscopy in dermatopathologic evaluation of skin tumors. JAMA Dermatol. 2016;152(3):312-317.

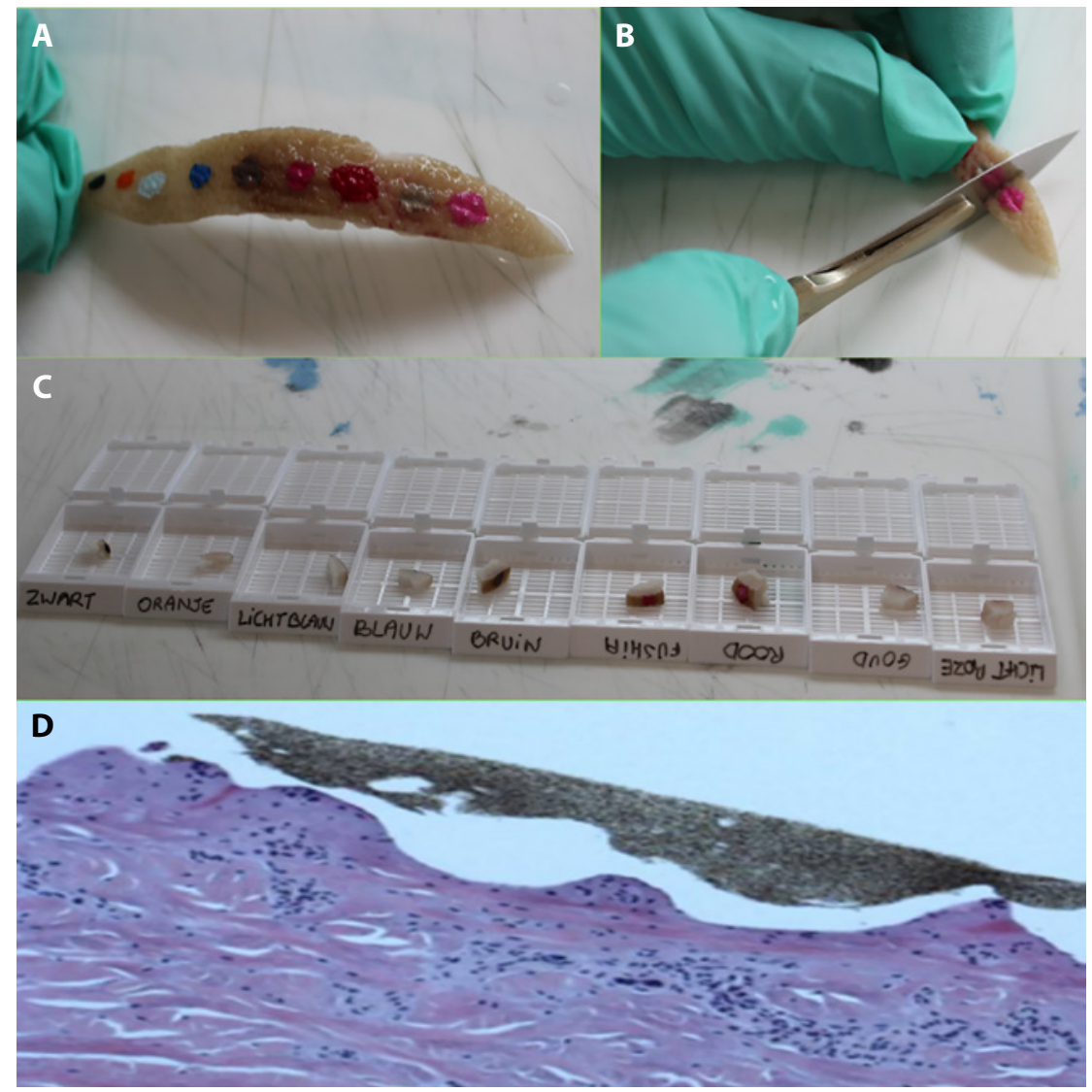

Figure 1. Pig skin with different nail polish drops (A). Preparing for routine processing (B, C). View under the microscope after routine processing, with a clearly visible dot on top, without damaging the underlying skin (D). [Copyright: (C2019 Lanssens et al.]
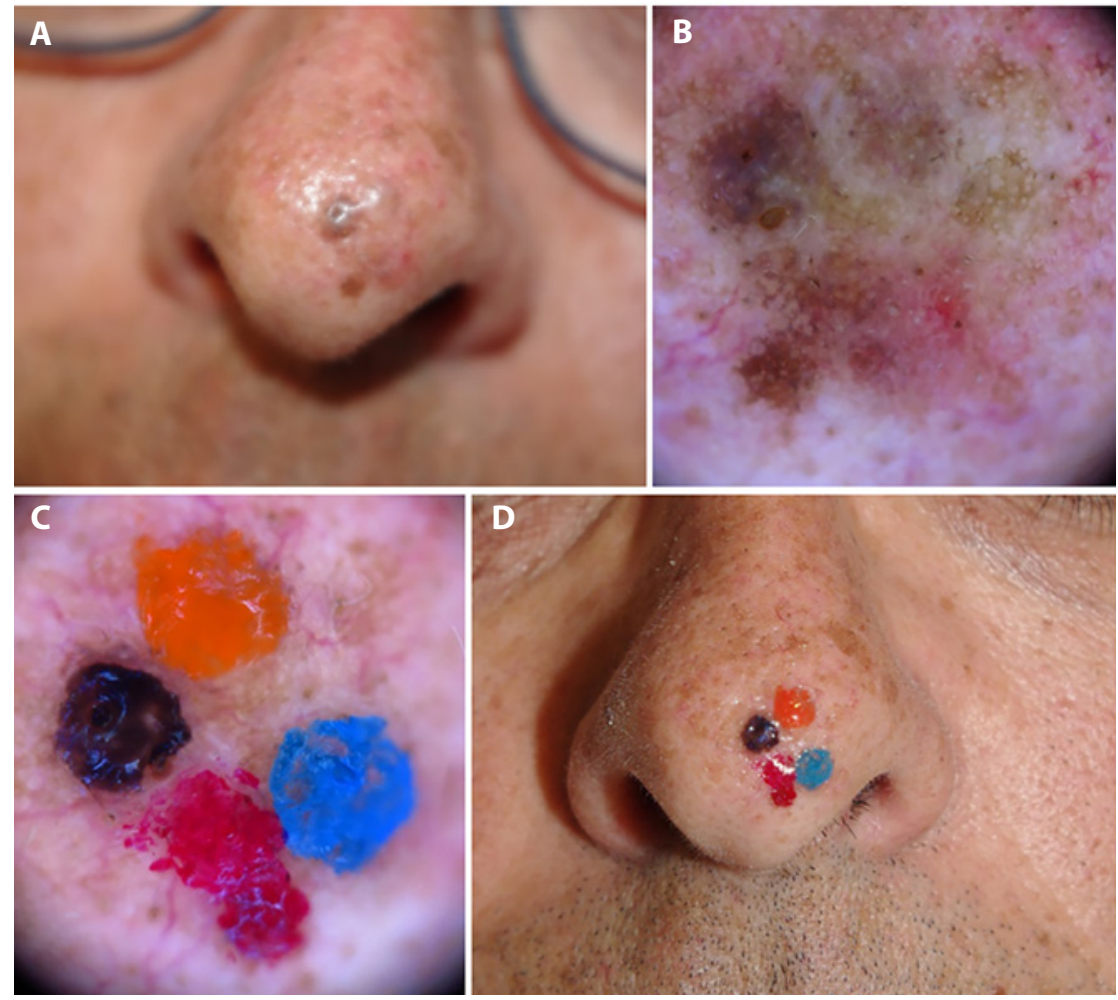

Figure 2. Clinical view of lesion on the nose (A). Dermoscopic view of the same lesion, composed of potential collision of 4 different lesions (B). Dotting of the 4 different areas in 4 different colors (C). Clinical view after dotting the lesion and before excision (D). [Copyright: (C2019 Lanssens et al.] 